\title{
Communication Recovery with Emergency Aerial Networks
}

\author{
Prodromos-Vasileios Mekikis, Student Member, IEEE, Angelos Antonopoulos, Senior Member, IEEE \\ Elli Kartsakli, Senior Member, IEEE, Luis Alonso, Senior Member, IEEE, and Christos Verikoukis, \\ Senior Member, IEEE
}

\begin{abstract}
In spite of the significant advancements in wireless connectivity, the static form of the network infrastructure cannot guarantee an uninterrupted operation of the ever-growing wireless consumer electronics in emergency situations such as natural disasters. In such occasions, employing flexible aerial nodes can tackle this issue by recovering the communication rapidly, when the need for connectivity is of utmost importance. In this paper, we study the use of aerial nodes for communication recovery after a communication breakdown. We provide an analytical model of the recovery probability that demonstrates the capabilities of such networks. In the performance evaluation, we show the effects of the altitude and the distance between the aerial nodes on the recovery probability and verify them with simulations. Moreover, we introduce our testbed and preliminary experimental work that shows promising results for aerial networks. Finally, we discuss useful insights for the network design and present some open issues that exist in this field.
\end{abstract}

Index Terms-Aerial Relay Nodes, Network Recovery, D2D Relaying, Coverage Probability, Testbed, Stochastic Geometry

\section{INTRODUCTION}

$\mathrm{O}$ VER the last few decades, the telecommunication industry has witnessed an unprecedented growth with a significant effect in our everyday life [1]. Recent advancements in 5G networks have continued this surge by introducing even more reliable and fast services to the consumers. However, in spite of the massive development of consumer electronics that require wireless connectivity (i.e., smartphones, smart home or health sensors, etc. [2], [3]), the static form of the current wireless telecommunications infrastructure poses threats and it is a critical barrier for the network reliability under certain conditions, e.g., after natural disasters like earthquakes, tsunamis and hurricanes, among others.

In such cases, except for the obvious immediate confusion in

A preliminary version of this paper has been submitted to the 2016 IEEE $5^{\text {th }}$ Global Conference on Consumer Electronics.

P.-V. Mekikis is with the University of Athens (UoA), Greece, and the Department of Signal Theory and Communications (TSC), Technical University of Catalonia (UPC), Spain, (e-mail: vmekikis@tsc.upc.edu).

A. Antonopoulos and C. Verikoukis are with the Telecommunications Technological Centre of Catalonia (CTTC/CERCA), Spain, (e-mail: \{aantonopoulos, cveri\}@cttc.es).

Elli Kartsakli is with Iquadrat Informatica S.L., Barcelona, Spain, (e-mail: ellik@iquadrat.com).

L. Alonso is with TSC, UPC, Spain, (e-mail: luisg@tsc.upc.edu). the society, there are also threatening side-effects caused by the communication failure. For instance, after the Nepal earthquake in 2015, "damaged communications infrastructure posed substantial challenges to rescue efforts [4]". Similarly, in Japan, one of the most technologically advanced countries in the world, the 2011 Tsunami caused a "communication breakdown [5]" for several days in the affected area, while after the Katrina hurricane in 2005 at New Orleans, USA, the city was "paralyzed by poor communications [6]". From the aforementioned cases, it can be seen that in many unfortunate events around the world, the damaged communication infrastructure on the ground may cause significant problems to the management of the crisis from the respective authority, e.g., the emergency operation center (EOC).

In order to aid the rescue efforts and recover partially the connectivity, device-to-device (D2D) communication can provide an ad-hoc solution to the network breakdown by connecting people without the need of a core network. However, the range of the D2D-enabled devices is not always enough to guarantee reliable communication [7]. Therefore, given the damaged core network, the deployment of flexible relay nodes is essential to recover the communication and expand the range of the $\mathrm{D} 2 \mathrm{D}$ devices by relaying their messages. These nodes should be able to deploy quickly at the places of interest, i.e., where rescue efforts take place, and to relocate according to the EOC needs.

In contrast to the inconvenient deployment of dynamic infrastructure on land, flexible aerial relay (FAR) nodes could be effective even in remote and unreachable areas [8]. These nodes can carry telecommunication and other kind of equipment (e.g., camera, sensors, etc.) and hover above the affected area for communication recovery and surveillance. There are several works that employ aerial nodes to provide connectivity over an area. The coverage and rate of a network with aerial base stations are studied in [9], where the authors optimize the altitude and transmission power of the aerial nodes and provide insightful results. Similarly, in [10], a drone small cell network is studied in terms of coverage and throughput to provide the optimal density, which is a crucial issue in aerial networks. However, both aforementioned works assume a combination of an aerial with a ground cellular network, which may not be a realistic scenario in emergency disaster scenarios. Moreover, in [11], the authors study the performance of an 


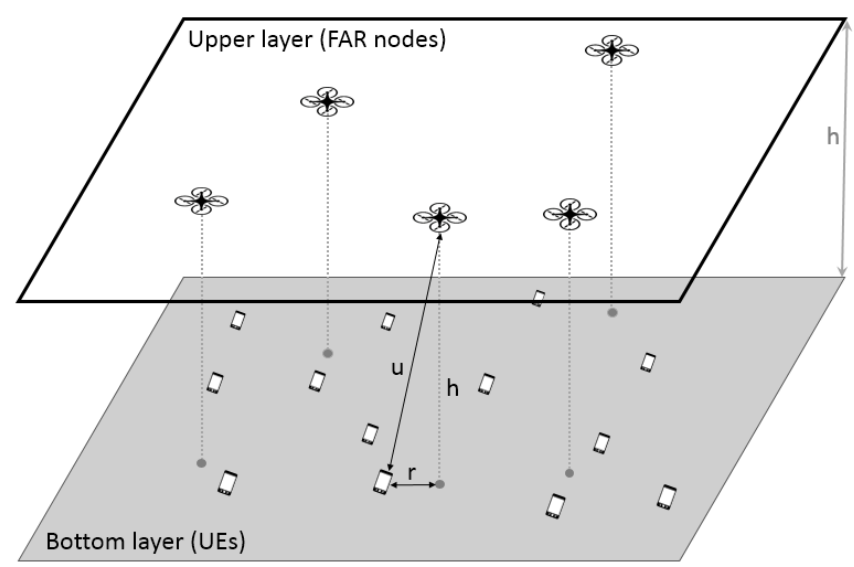

Fig. 1. Network topology

aerial network modeled as a binomial point process and present interesting results regarding the downlink coverage probability for different aerial node altitudes. However, there is no work that studies the recovery probability of an aerial network deployed during the first hours of a crisis to support the rescue efforts, while being reliable without any assistance from the network in the ground.

In this paper, we study the probability of communication recovery in a network using FAR nodes. The nodes assist the rescue efforts in two ways: i) they provide communication between the user equipment (UE) and the EOC that manages the crisis, and ii) they assist the D2D communication among the ground users, whenever possible. We model the network as a two-layer Poisson point process (2L-PPP), in which the two layers are separated by a certain distance $h$. The ground users are deployed as a PPP in the bottom layer, while the aerial nodes are deployed as a PPP in a plane over the ground user layer. Then, we derive mathematical formulas that provide the probability of correct communication. To that end, the main contributions of this paper are threefold:

- We mathematically derive the probability of successful connection between a ground node and the nearest FAR, i.e., the probability that the network has recovered the communication.

- We derive the recovery probability, i.e., the probability that the network has recovered the communication via the EOC and the FAR nodes.

- We present some preliminary experimental results obtained through a real life testbed.

- We identify and discuss several open issues that need to be considered in future aerial networks.

The rest of the paper is organized as follows. The system model is described in Section II. The mathematical analysis is presented in Section III and the numerical results in Section IV. The testbed and our preliminary experimental work is described in Section V, while the open issues of aerial networks are presented in Section VI. Finally, Section VII concludes the paper.

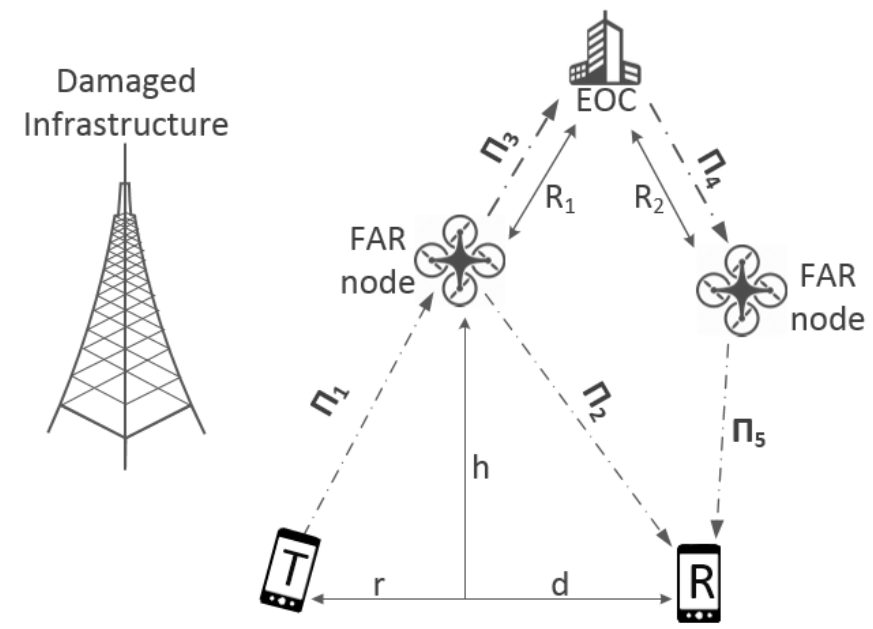

Fig. 2. Communication model (UE-T $\rightarrow$ UE-R): i) Directly via FAR node ii) Via FAR nodes and EOC.

\section{SYSTEM MODEL}

As shown in Fig. 1, we consider a network of UEs that are represented by a Poisson point process (PPP) $\Phi_{U}=$ $\left\{x_{1}, x_{2}, x_{3}, \ldots\right\}$ with intensity $\lambda$, where $x_{i}, \forall i \in \mathbb{N}$, denotes the locations of the UEs on the plane. The network infrastructure is assumed to be defective/damaged and, thus, FAR nodes are deployed above the UEs (i.e., affected area), hovering at an altitude of $h \mathrm{~m}$. Thus, the FAR nodes are represented as a second PPP layer with $\Phi_{F}=\left\{y_{1}, y_{2}, y_{3}, \ldots\right\}$ and intensity $\lambda_{F}$, where $y_{j}, \forall j \in \mathbb{N}$, denotes the locations of the FAR nodes at the upper layer, as shown in Fig. 1. Furthermore, an EOC that manages the rescue efforts is located at a distance $R$ from the center of the affected area and is equipped with a transceiver for communicating with the FAR nodes.

In our study, we assume that a transmitting UE, denoted as UE-T, attempts communication with another UE, denoted as UE-R, either without or with the need of the EOC, as shown in Fig. 2. In the first case, UE-T transmits its data successfully to the nearest FAR node (with probability $\Pi_{1}$ ) at a distance $u=$ $\sqrt{r^{2}+h^{2}}$, where $\mathrm{r}$ is the distance between the projection of the nearest FAR node to the UE layer and the UE. Then, the FAR node delivers the message to the recipient UE-R located at a distance $\sqrt{d^{2}+h^{2}}$ from the FAR node (with probability $\Pi_{2}$ ). However, for diversity reasons, the FAR node transmits these data to the EOC (with success probability $\Pi_{3}$ ). Then, the EOC delivers the message to the nearest FAR node of UE-R (probability $\Pi_{4}$ ) and, finally, UE-R receives the packet from its nearest FAR (probability $\Pi_{5}$ ). In this way, the communication is recovered between the two UEs.

Moreover, we examine the ability of FARs, UEs or EOC to decode successfully a message based on the received power denoted as $P_{r x}=P_{t x} g u^{-\alpha}$, where $P_{t x}$ is the transmission power (i.e., $P_{E O C}$ for EOC and $P$ for UE/FAR), $u$ is the transmission distance, $\alpha$ is the path loss exponent and $g$ is the fast fading power coefficient, a random variable that is independent and identically distributed (i.i.d.). As a result, the amplitude fading $\sqrt{g}$ is Rayleigh distributed with a scale parameter $\sigma=1$, thus 
$g$ is exponentially distributed with mean value $\mu=1$. Each FAR node experiences interference from the other active mobile devices. Therefore, a mobile device is considered connected to its nearest FAR (i.e., is able to deliver a message), when the received signal to noise plus interference ratio (SINR) is higher than a threshold $\gamma$, as it is given in

$$
\operatorname{SINR}=\frac{P_{t x} g u^{-\alpha}}{W+\sum_{x_{i} \epsilon \Phi_{u}} P_{t x} g_{i} u_{i}^{-\alpha}} \geq \gamma
$$

where $W$ is the additive white Gaussian noise power, modeled as a constant zero mean Gaussian Random Variable (RV). The same formula applies to the communication between FARs and the EOC. However, it should be mentioned that, since there is one EOC, the FAR nodes do not perceive interference during the message reception at $\Pi_{4}$.

\section{RECOVERY PROBABILITY ANALYSIS}

In this section, we derive the probability that FAR nodes recover the communication in the affected area. In the beginning, we study the direct communication between the UE and the FAR node, and then the communication between the FAR nodes and the EOC. In the end, we combine our results to provide the general recovery probability of the emergency network.

\section{A. UE-FAR node communication $\left(\Pi_{1}, \Pi_{5}\right)$}

In order to calculate the probability that the UE is able to transmit successfully its data to the nearest FAR node, we have to consider (1). In this formula, the distance $u$ between the UE and FAR is affected by the height of the FAR and it is given by $u=\sqrt{h^{2}+r^{2}}$. Then, we calculate the probability of successful connection $\left(\Pi_{1}\right)$ with the FAR node, which is given by the following Lemma.

Lemma 1: The probability of successful connection between the UE-T and the FAR node is given by the following formula

$$
\Pi_{1}=\int_{0}^{\infty} 2 \pi \lambda r e^{-\pi \lambda r^{2}-\pi \lambda\left(h^{2}+r^{2}\right) \varrho} e^{-\gamma W\left(h^{2}+r^{2}\right)^{a / 2}} d r,
$$

where

$$
\varrho=\gamma^{2 / a} \int_{\gamma^{-2 / a}}^{\infty} \frac{1}{1+k^{a / 2}} d k .
$$

Proof: The proof is provided in Appendix A.

Similar approach has to be followed in order to calculate the probability $\Pi_{5}$. However, in this case, the interference is caused by the PPP of the FAR nodes. Therefore, the probability $\Pi_{5}$ is given by

$$
\Pi_{5}=\int_{0}^{\infty} 2 \pi \lambda r e^{-\pi \lambda r^{2}-\pi \lambda_{F}\left(h^{2}+r^{2}\right) \varrho} e^{-\gamma W\left(h^{2}+r^{2}\right)^{a / 2}} d r,
$$

which is similar to $\Pi_{1}$, but the intensity of the interferers is $\lambda_{F}$.

\section{B. FAR node-UE communication $\left(\Pi_{2}\right)$}

In the case of probability $\Pi_{2}$, there is no need to condition on the nearest UE, as it is located at a fixed distance $\sqrt{h^{2}+d^{2}}$ from the FAR node. Therefore, the formula that provides probability $\Pi_{2}$ is given by the following lemma.

Lemma 2: The probability of successful connection between the FAR node and the UE-R is given by the following formula

$$
\Pi_{2}=e^{-\gamma W\left(h^{2}+d^{2}\right)^{a / 2}} e^{-\pi \lambda \gamma^{\frac{2}{a}}\left(h^{2}+d^{2}\right) \Gamma\left(1+\frac{2}{a}\right) \Gamma\left(1-\frac{2}{a}\right),}
$$

where $\Gamma$ is the gamma function and it is given by

$$
\Gamma(z)=\int_{0}^{\infty} x^{z-1} e^{-x} d x .
$$

Proof: The proof is provided in Appendix B.

\section{FAR-EOC communication $\left(\Pi_{3}\right)$}

Next, we calculate the probability $\Pi_{3}$, that a FAR node is able to deliver its messages to the EOC. As we have already explained, the EOC is located at a distance $R$ from the center of the affected area. Therefore, from the EOC point of view, the affected area acts as a cluster at a distance $\mathrm{R}$ and, thus, we assume that the interference at the EOC is originated from a cluster at a distance $R_{1}$. Hence, the probability $\Pi_{3}$ is given in the following lemma.

Lemma 3: The probability of successful connection between the FAR node and the EOC is given by the following formula

$$
\Pi_{3}=e^{-\gamma W R_{1}{ }^{a}} e^{-\pi \lambda_{c} \gamma^{\frac{2}{a}} R_{1}{ }^{2} \frac{2 \pi / a}{\sin (2 \pi / a)}} .
$$

Proof: The proof is provided in Appendix C.

\section{EOC-FAR communication $\left(\Pi_{4}\right)$}

The probability $\Pi_{4}$ can be easily derived as there is no interference, i.e., there is only one EOC. To that end, we have to calculate the probability of successful communication at a distance $R_{2}$, which is given by the following lemma.

Lemma 4: The probability of successful connection between the EOC and the FAR node is given by the following formula

$$
\Pi_{4}=e^{-\frac{\gamma W R_{2}{ }^{a}}{P_{E O C}}}
$$

Proof: In this case, we have to calculate the probability that the signal to noise ratio (SNR) is higher than a threshold $\gamma$. This can be accomplished as follows:

$$
\begin{aligned}
& \Pi_{4}(S N R>\gamma)=\Pi_{4}\left(P_{E O C} h R_{2}^{-a}>\gamma W\right)= \\
& =\Pi_{4}\left(h>\frac{\gamma W}{P_{E O C} R_{2}^{-a}}\right) \Rightarrow \\
& \stackrel{(i)}{\Rightarrow} \Pi_{4}=e^{-\frac{\gamma W R_{2}}{P_{E O C}},}
\end{aligned}
$$


TABLE I

SIMULATION PARAMETERS

\begin{tabular}{|c|c|c|}
\hline Symbol & Parameter & Values \\
\hline$a$ & Path loss exponent & $2.2-4$ \\
\hline$R_{1}$ & Distance FAR1-EOC & $250-1000 \mathrm{~m}$ \\
\hline$R_{2}$ & Distance FAR2-EOC & $500 \mathrm{~m}$ \\
\hline$A$ & Simulation area & $3000 \mathrm{~m} \times 3000 \mathrm{~m}$ \\
\hline$W$ & Noise & $10^{-7} \mathrm{~W}$ \\
\hline$\gamma$ & Threshold & $-10-20 \mathrm{~dB}$ \\
\hline$h$ & FAR altitude & $0-70 \mathrm{~m}$ \\
\hline$d$ & Distance FAR node - UE-R & $0-60 \mathrm{~m}$ \\
\hline$P_{E O C}$ & EOC transmission power & $4 \mathrm{~W}$ \\
\hline$P_{F}$ & FAR/UE transmission power & $1 \mathrm{~W}$ \\
\hline$\lambda_{c}$ & Cluster intensity & 1 per total simulation area \\
\hline$\lambda$ & UE intensity & $10^{-3}$ UEs per $\mathrm{m}^{2}$ \\
\hline$\lambda_{F}$ & FAR intensity & $10^{-4}$ FAR nodes per $\mathrm{m}^{2}$ \\
\hline
\end{tabular}

where $(i)$ follows from the exponential distribution of the Rayleigh fading, and $P_{E O C}$ is the transmission power of the EOC transceiver.

\section{E. Recovery probability}

Since we have derived all probabilities, we combine them to calculate the recovery probability of the emergency aerial network. A UE attempts to communicate with another device through a FAR node directly and, if this is not possible, then its data are redirected through the EOC and the nearest FAR node of the recipient device. To that end, since all probabilities are independent, the recovery probability $\Pi_{\mathrm{R}}$ is given by the following formula

$$
\Pi_{\mathrm{R}}=\underbrace{\Pi_{1} \Pi_{2}}_{\text {FAR }}+\underbrace{\left(1-\Pi_{2}\right) \Pi_{1} \Pi_{3} \Pi_{4} \Pi_{5}}_{\text {FAR and EOC }} \text {. }
$$

By replacing the probabilities in $\Pi_{1}$ from (3), $\Pi_{2}$ from (6), $\Pi_{3}$ from (8), $\Pi_{4}$ from (9), and $\Pi_{5}$ from (5), we obtain the final result. Note that the probability $\Pi_{5}$ is the same with $\Pi_{1}$.

\section{Performance Evaluation}

In this section, we validate the proposed theoretical framework via extensive simulations and provide useful insights on the use of FAR nodes for the network recovery. For our simulations, we employ a custom-made simulator developed in the computing environment Matlab R2014a.

\section{A. Simulation Setup}

The topology of the simulated scenario is depicted in Fig. 1. Unless otherwise stated, the decoding threshold $\gamma$ is assumed fixed at $-10 \mathrm{~dB}$. Moreover, we assume that the FAR nodes and the UEs of the rescue teams have an isotropic antenna with a transmission power of $1 \mathrm{~W}$, respecting the limits of unlicensed ISM bands by the Federal Communications Commission (FCC) [12]. Similarly, the transmission power of the EOC is $1 \mathrm{~W}$, but with an antenna gain of $6 \mathrm{dBi}$. To that end, the Effective Isotropically Radiated Power (EIRP) is $36 \mathrm{dBm}$ or $4 \mathrm{~W}$. Additionally, the path loss exponent is fixed at 4 for the communication between the FAR nodes and the UEs (assuming urban environment), and it is set at 2.2 for the line-of-sight

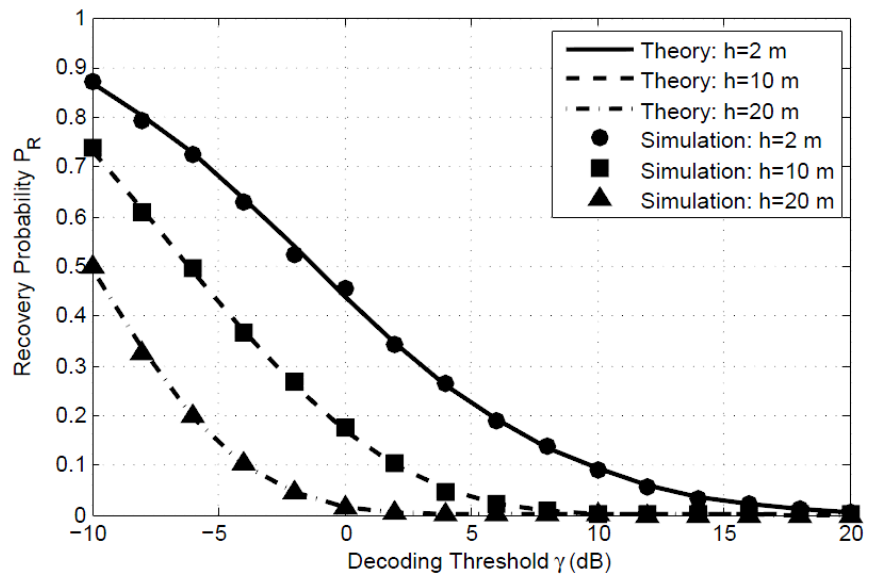

Fig. 3. Recovery probability $P_{R}$ for different values of decoding threshold

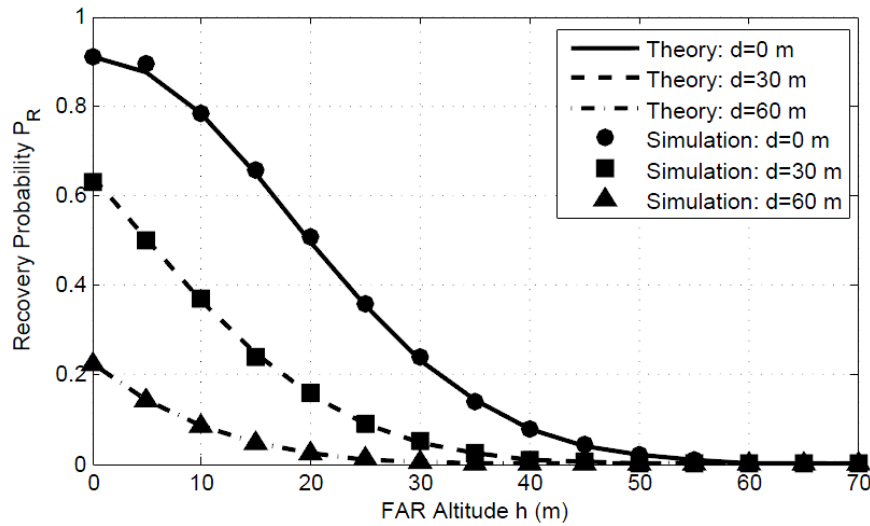

(a) Through FAR node directly $\left(\Pi_{1} \Pi_{2}\right)$

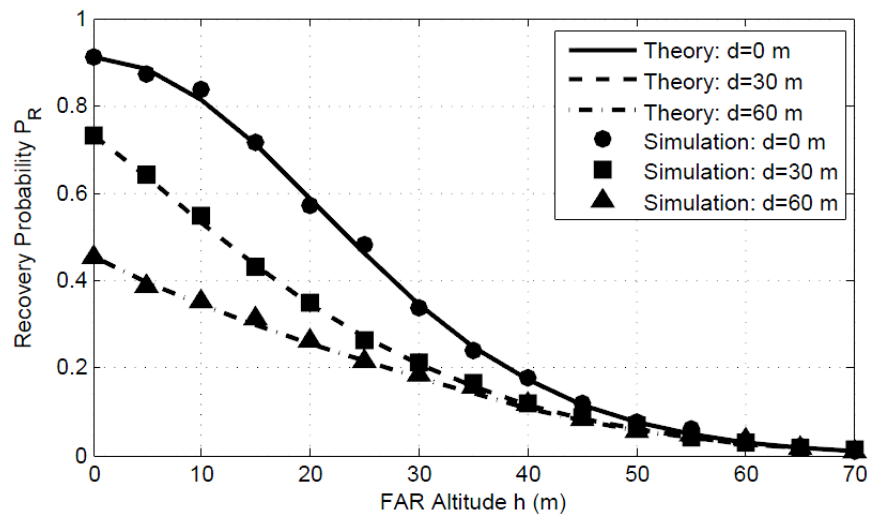

(b) Through FAR and EOC $\left(\Pi_{\mathrm{R}}\right)$

Fig. 4. Recovery probability $P_{R}$ for different values of FAR altitude and distance $d$. (a) Communication through FAR node only, (b) Communication through FAR and EOC.

communication between the EOC and the FAR nodes. The distance between the FAR node and the recipient UE varies between 0 and $60 \mathrm{~m}$ and the altitude of the FAR node between 0 and $70 \mathrm{~m}$. Finally, we assume one affected area in the simulation area that has an intensity of $10^{-3}$ UEs per $\mathrm{m}^{2}$ and $10^{-4}$ FAR nodes per $\mathrm{m}^{2}$. All parameters are listed in Table I.

\section{B. Analytical and Simulation Results}

In this section, we validate the recovery probability and 


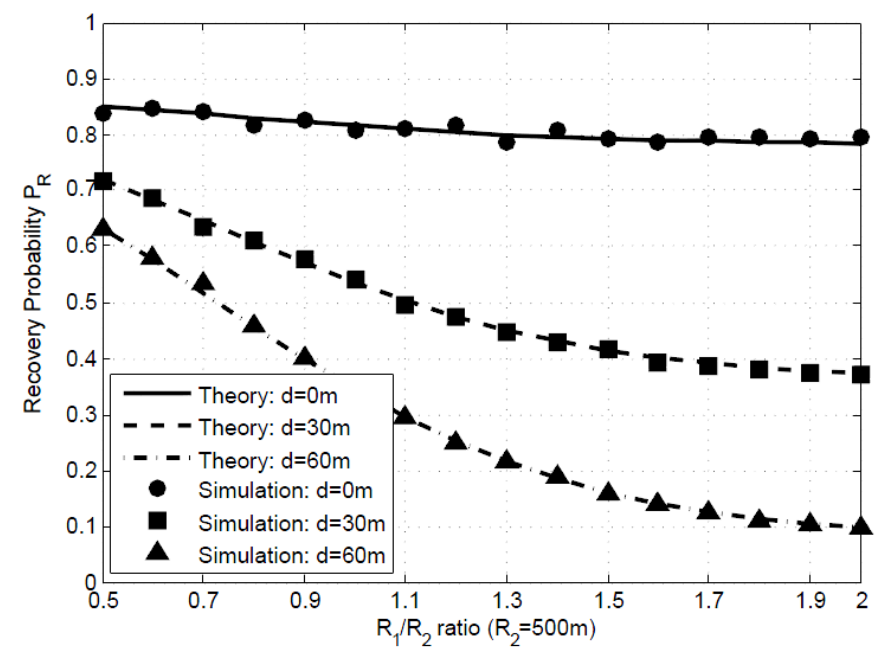

Fig. 5. Recovery probability $\mathrm{P}_{\mathrm{R}}$ for various values for the $R_{1} / R_{2}$ ratio

derive information regarding the performance of the emergency aerial network. In Fig. 3, we present the recovery probability versus the decoding threshold for different values of the FAR altitude. As it can be seen, the simulation follows the theory tightly and, as expected, the recovery probability becomes lower as the decoding threshold or the FAR altitude increases. Obviously, as the threshold increases, it is more probable for the receiver to fail during the decoding. Similarly, for higher altitudes, the path loss between the transmitter and the receiver increases resulting in lower SINR.

In Fig. 4, we provide a more descriptive evaluation of the effects caused by increasing the altitude and the $d$-distance. To highlight the benefits of employing the EOC route in the network model, we demonstrate the network performance without and with an EOC in Fig. 4a and Fig. 4b, respectively. As we can observe, increasing the $d$-distance or the altitude decreases the recovery probability in both cases, due to the higher path loss between the FAR node and the UE. However, the drop is not as severe in Fig. 4b, especially for high values of the $d$-distance. For instance, at $d=60 \mathrm{~m}$ and $h=10 \mathrm{~m}$, the recovery probability is approximately $8 \%$ in Fig. $4 \mathrm{a}$, while in Fig. 4 b, it is approximately $36 \%$. This stems from the fact that for high $d$-distances, the FAR node is not able to communicate effectively with the UEs and, thus, routing the messages via the EOC can provide significant benefits. To that end, there is an alternative path when the distance between the two UEs is very large, which is a crucial characteristic for emergency networks.

Another important issue that needs to be evaluated in emergency networks is the distance between the affected area and the EOC. Therefore, in Fig. 5, we present the effects of the changes in the distance $R_{1}$ between the FAR node and the EOC on the recovery probability, while keeping the distance $R_{2}$ fixed at $500 \mathrm{~m}$ and the $d$-distance at 0,30 , and $60 \mathrm{~m}$. As expected, by increasing the distance $R_{1}$, we notice a drop in the recovery probability, which is steeper for higher values of the $d$-distance. This is due to the combination of the increased $d$-distance with the increased distance $R_{1}$ that compromises both the communication through the FAR nodes and the communication via the EOC. Therefore, in order to minimize the performance drop in cases where both the $d$-distance and the $R$-distance is

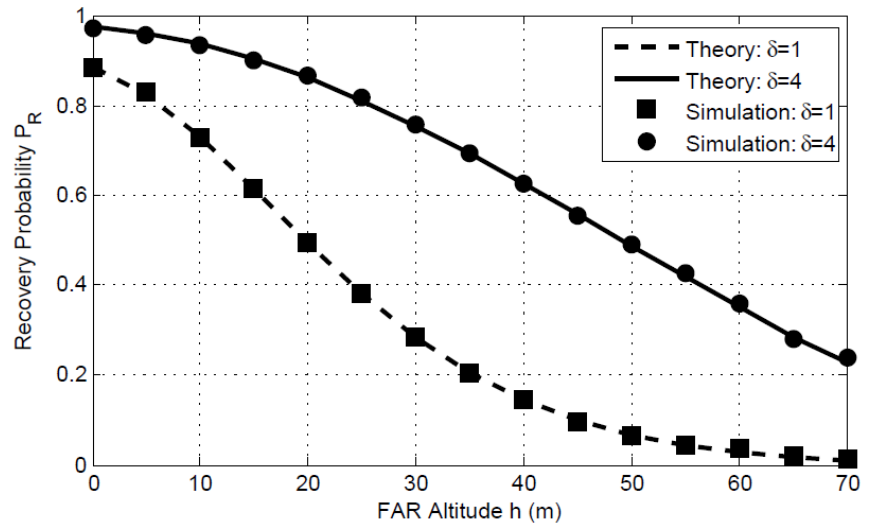

Fig. 6. Recovery probability $P_{R}$ Vs. FAR altitude for different frequency reuse factors.

high, i.e., high path loss, it is needed to employ methods that eliminate the interference, which is the other major limiting factor of the communication.

To that end, in Fig. 6, we demonstrate the effects of using frequency reuse on the recovery probability for different FAR altitude values. With frequency reuse, the reuse factor $\delta \geq 1$, determines the number of different frequency bands employed by the network [13]. It is worth mentioning that although this technique can reduce the interference drastically, it drops the throughput of the system. However, in emergency networks where reliability is vital, employing short messages for the communication of important matters, e.g., need for ambulance or rescue teams in certain areas, etc., can provide the necessary information without the need for high throughput. As we can see in Fig. 6, the recovery probability for $\delta=4$ reaches $75 \%$ for $h=30 \mathrm{~m}$, which is a boost of approximately $200 \%$ compared to the same case for $\delta=1$.

\section{EXPERIMENTAL RESULTS}

In order to support our idea and present some preliminary experimental results on the performance of aerial networks, we have created a proof-of-concept testbed that incorporates a FAR node, an EOC and UEs. The testbed imitates a critical situation, in which the communication should be recovered, in order to avoid a communication breakdown. In the following, we explain the different parts of the testbed and show its performance.

\section{A. Implementation}

Our implementation is depicted in Fig. 7. For the communication equipment, we have employed four CrossBow TelosB nodes. The operating system of these devices is the TinyOS and they are programmed with the NesC programming language. Furthermore, we have programmed three different kinds of nodes:

a) Two of these devices act as UEs that rescue teams or survivors employ for communication using short preset messages. These nodes are programmed to send a message to the EOC and another rescue team periodically.

b) The relay that is located on the FAR node and aids the exchange of the messages between the rescue teams and the EOC. 


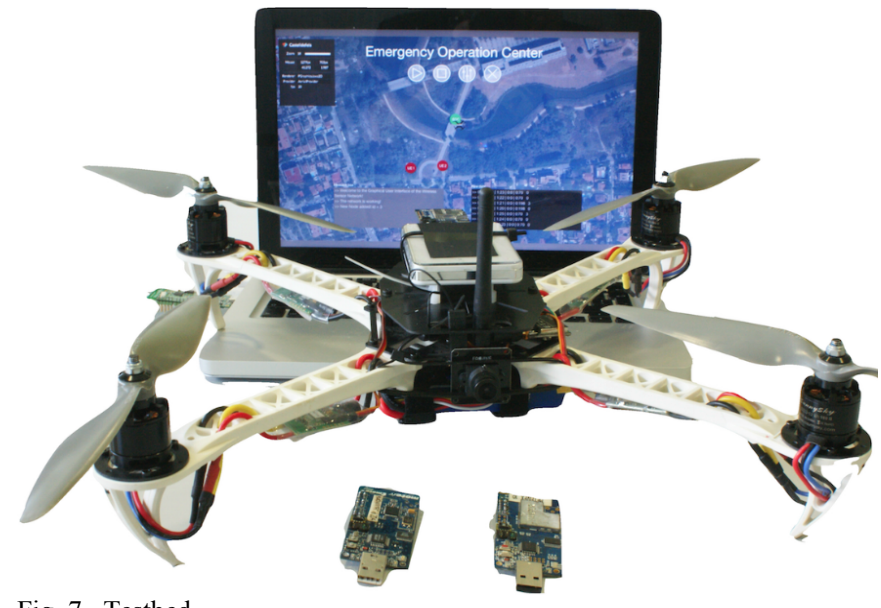

Fig. 7. Testbed.

c) The EOC node that receives all the messages from the rescue teams through the FAR node and displays them to a graphical user interface (GUI). Moreover, it is able to transmit similar messages to the rescue team UEs.

Moreover, we have assembled a custom-made quadcopter that carries the relay node and a video camera for the surveillance of the affected area. Also, the status of the network and the management of the FAR nodes is handled by the EOC GUI. The GUI was programmed in Processing IDE using opensource libraries (i.e., ControlP5 and UnfoldingMaps).

As shown in Fig. 8, in the main screen it presents an interactive satellite map that is overlaid by figures that mark the location of the EOC, the UEs and the FAR node. Information regarding the FAR node and UE status are shown when the user moves the pointer over the respective figure. Moreover, a UE is shown as a red circle when there is no established link between the EOC and the UE (see Fig. 8a). On the other hand, a UE turns green when the EOC is able to communicate with it through the FAR, as shown in Fig. 8b.

\section{B. Experiment}

The goal in our experiment scenario is to establish a connection between the EOC and the UEs though the FAR node, in order to achieve a quick communication recovery and be able to supervise and manage the operation though the GUI. Indeed, the system works as expected at all times by providing fast communication between the EOC and the UEs.

In Fig. 9, we demonstrate the results on the network recovery versus the FAR altitude after extensive experiments. In contrast to the results of our simulation analysis, it can be seen that the performance of the experimental network is excellent even for high altitudes, i.e., at $100 \mathrm{~m}$ the network recovers the communication with probability over $80 \%$. This stems from the fact that in the experimental network there is not any substantial interference from the nodes. On the contrary, in our simulations, we assume a worst case analysis in which the interference is significant and there is not line-of-sight. All things considered, both the experimental and the simulation results are promising and prove that aerial networks can contribute substantially in emergency situations.

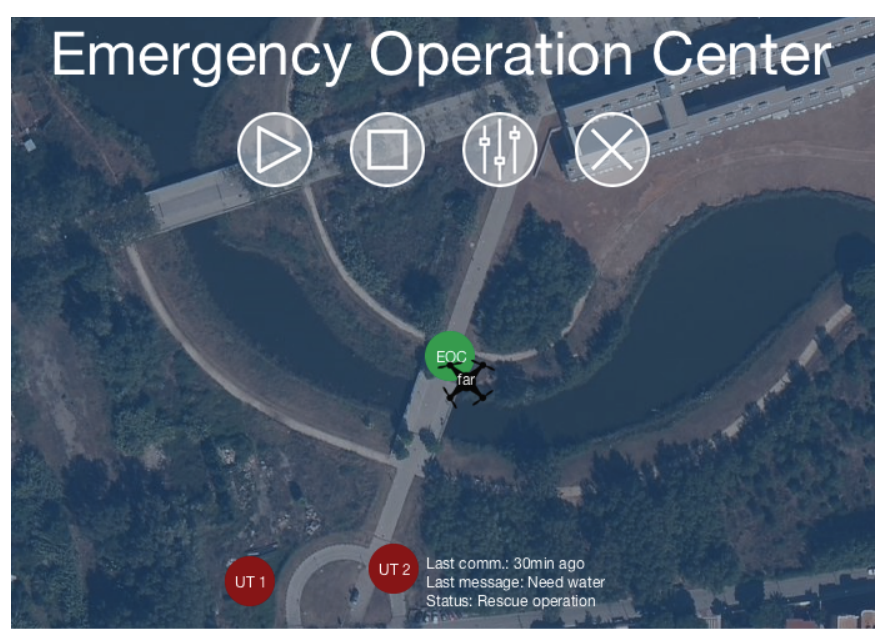

(a) UEs are not able to communicate with the EOC.

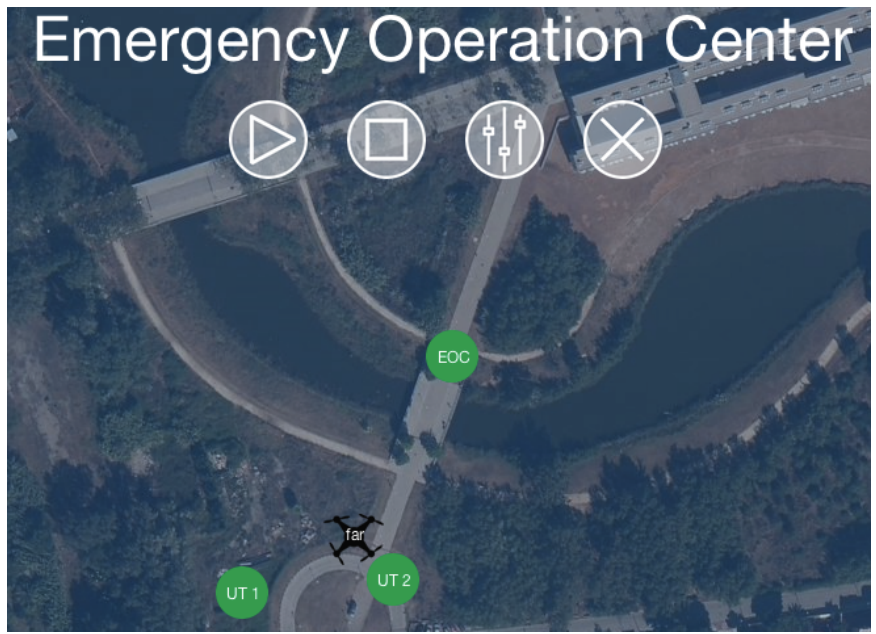

(b) UEs are connected with the EOC and with each other.

Fig. 8. Screenshots of the main GUI screen.

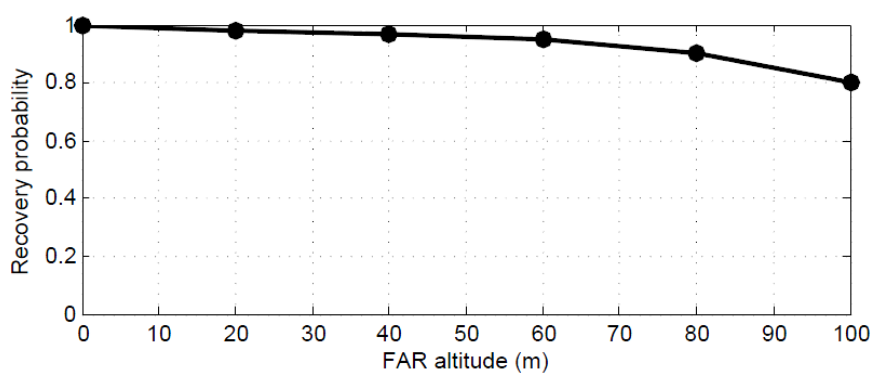

Fig. 9. Experimental results.

\section{OPEN ISSUES}

Although employing aerial nodes for communication recovery appears as a promising solution for emergency situations and standard cellular networking, there are still various open issues that should be considered in the future. In the following, we present a list of open issues and propose possible solutions. 


\section{A. Energy Supply}

The energy supply is an important issue for FAR nodes, as their lifetime is short due to a combination of the high energy consumption of the motors that provide thrust to the aerial node and the constrained load-lifting ability for higher capacity and, thus, heavier batteries [15]. To that end, the FAR nodes are not able to sustain a stable recovery for more than an hour according to current standards. However, there are two solutions that could alleviate the energy limitations while providing similar communication performance to our proposal.

One solution could be to equip the EOC with a secondary FAR node for every primary FAR node. In this way, when the battery of the primary FAR reaches a critical level, a second FAR will be deployed to replace it using seamless handover to sustain the communication without any disruption.

Another approach to support FAR use in urban scenarios could be the use of docking posts for the FAR nodes. The docking posts are deployed in random locations around the city and will be equipped with solar harvesting panels for recharging the FAR. In this way, it is possible to solve the energy limitation issue in a green way, while providing connectivity in predetermined areas.

\section{B. Wireless Backhaul/Fronthaul}

In our work, the communication between FARs and the EOC is direct without any intermediate node. However, in cases where the EOC is located out of the FAR node range, there is a need for a more sophisticated solution [16]. In such cases, routing the traffic through aerial nodes positioned between FARs and the EOC and act as relays could solve this issue. Moreover, in regular cellular networks, aerial nodes should be able to deliver the traffic to the core network. To achieve optimal performance, new protocols have to be proposed that take into account the peculiarities of aerial networks.

\section{Self-organization}

FAR nodes with the ability to self-organize with optimal placement, seamless handover and load balancing would improve drastically the network performance [17]. In this way, the aerial nodes would be utilized more efficiently in cases of unbalanced traffic distribution in the different places of the affected area.

\section{Weather conditions}

The weather conditions play a significant role for the normal operation of an aerial network and affect its stability, e.g., in case of strong wind conditions. This is the reason that various works suggest a high altitude placement of the aerial nodes [18]. However, in high altitudes, the communication performance of the network drops as the path loss and signal fading is increased. The docking posts explained in Section VI-A could solve this issue in regular non-emergency aerial networks.

\section{E. Proactive Caching}

Caching data proactively on aerial nodes is an effective technique for aerial networks, especially in emergency situations. For instance, it is probable that most users would want to get informed regarding the current situation of the crisis, to have maps with help points and medical stations, etc. Proactively caching content on the aerial nodes regarding this event would greatly increase the performance of the network and provide better quality of service to the users [19].

\section{F. Blockage Effects}

Another important issue that is closely related to the aerial networks is the effects of the blockages from buildings, trees, etc. in the communication performance. There are some works that consider blockages in their system model by taking into account urban statistical parameters for the ratio of the built-up area to the total area, the mean number of urban structures per unit area, etc. [9]. However, this is an abstract handling and it can be done with a more concrete and accurate procedure, as in [20], using a mathematical framework to model blockages with random sizes, locations, and orientations in cellular networks using concepts from random shape theory. In this way, the results will be more realistic, which is significant for emergency networks. Finally, it is worth noting that by considering blockages in the mathematical modeling of the network, the effects of the altitude changes will not be so severe, as the increase in the path loss will be counterbalanced by the elimination of the blockages for higher altitudes.

\section{CONCLUSION}

Emergency flexible aerial relay nodes can recover the communication after natural disasters, when the need for reliable connectivity is of utmost importance. In this paper, we study the use of aerial nodes for the communication recovery and D2D relaying between users. We have derived the probability of successful recovery and presented the performance of our network by validating through simulations. To support our work, we have implemented a testbed to demonstrate the abilities of an aerial network. The preliminary experimental work demonstrates potential for a reliable solution in emergency situations. Moreover, we provide useful insights and open issues that will further expand the research in this significant area. As a future work, we plan to discover the possibility of employing self-organizing UAVs to cover the connectivity needs in traffic-congested places.

\section{ACKNOWLEDGMENT}

This work has been funded by AGAUR (2014-SGR-1551) and the research projects CellFive (TEC2014-60130-P), and SmartNRG (European Commission FP7-612294).

\section{APPENDIX A}

In this Appendix, we show the derivations of Lemma 1. We follow the derivations of [13] for the special case that the interferers are subject to Rayleigh fading, and that $u=$ $\sqrt{r^{2}+h^{2}}$ for the final result. To that end, the probability $\Pi_{1}$ can be obtained by conditioning to the projection of the nearest FAR to the bottom layer, which is at a distance $r$ from the UE. However, we have to take into account the altitude $h$ when we consider the path loss between the receiver and the transmitter or the interferers. Therefore, we obtain

$$
\Pi_{1}=\mathbb{E}_{r}[P(\operatorname{SINR}>\gamma \mid r)],
$$

Then, we can take the integral

$$
\Pi_{1}=\int_{0}^{\infty} P(\operatorname{SINR}>\gamma) f(r) d r,
$$


where $f(r)$ is the probability density function of the distance to the nearest FAR node and it is given by

$$
f(r)=2 \pi \lambda r e^{-\pi \lambda r^{2}}
$$

Thus,

$$
\Pi_{1}=\int_{0}^{\infty} 2 \pi \lambda r e^{-\pi \lambda r^{2}} P\left(h>\gamma u^{a}\left(W+I_{u}\right)\right) d r
$$

Since the Rayleigh fading coefficient $\mathrm{h}$ is exponentially distributed with mean value 1 , we obtain

$$
\Pi_{1}=\int_{0}^{\infty} 2 \pi \lambda r e^{-\pi \lambda r^{2}} e^{-\gamma W u^{a}} \mathcal{L}\left(\gamma u^{a}\right) d r .
$$

The Laplace transform of the interference is given by [13]

$$
\mathcal{L}\left(\gamma u^{a}\right)=e^{-\pi \lambda u^{2} \varrho}
$$

where

$$
\varrho=\gamma^{2 / a} \int_{\gamma^{-2 / a}}^{\infty} \frac{1}{1+k^{a / 2}} d k .
$$

To that end,

$$
\Pi_{1}=\int_{0}^{\infty} 2 \pi \lambda r e^{-\pi \lambda r^{2}} e^{-\pi \lambda u^{2} \varrho} e^{-\gamma W u^{a}} d r .
$$

However, we know that $u=\sqrt{r^{2}+h^{2}}$. Thus,

$$
\Pi_{1}=\int_{0}^{\infty} 2 \pi \lambda r e^{-\pi \lambda r^{2}} e^{-\pi \lambda\left(\sqrt{r^{2}+h^{2}}\right)^{2} \varrho} e^{-\gamma w\left(\sqrt{r^{2}+h^{2}}\right)^{a}} d r,
$$

which concludes the proof.

\section{APPENDIX B}

In this Appendix, we show the derivations of Lemma 2. In this case, the FAR node transmits to a fixed distance $d$. Therefore, according to [21], the success probability in a Poisson field of interferers of intensity $\lambda$ at a distance $d$ is given by

$$
\Pi_{2}=e^{-\gamma W u^{a}} e^{-\pi \lambda \gamma^{\frac{2}{a}} u^{2} \Gamma\left(1+\frac{2}{a}\right) \Gamma\left(1-\frac{2}{a}\right)} .
$$

By taking into account that $u=\sqrt{d^{2}+h^{2}}$, we obtain

$$
\Pi_{2}=e^{-\gamma W\left(\sqrt{d^{2}+h^{2}}\right)^{a}} e^{\left.-\pi \lambda \gamma^{\frac{2}{a}\left(\sqrt{d^{2}+h^{2}}\right.}\right)^{2} \Gamma\left(1+\frac{2}{a}\right) \Gamma\left(1-\frac{2}{a}\right),}
$$

which concludes the proof.

\section{APPENDIX C}

In this Appendix, we will show the derivations of Lemma 3. As we have already explained, the EOC is located at a distance $R$ from the center of the affected area. Therefore, from the EOC point of view, the affected area acts as a cluster at a distance $R$. To that end, to calculate the probability $\Pi_{3}$, we need to take the following steps

and, thus,

$$
\Pi_{3}=P\left(h>\gamma(W+I) R_{1}{ }^{a}\right)
$$

As we know that the fading is Rayleigh distributed, we get

$$
\Pi_{3}=e^{-\gamma R_{1}^{a}(W+I)}=e^{-\gamma R_{1}^{a} W} \mathcal{L}\left(\gamma R_{1}^{a}\right) .
$$

The lower bound of the inter-cluster interference is provided by [22] and it is given by

$$
\mathcal{L}\left(\gamma R_{1}^{a}\right)=e^{-\pi \lambda_{c} \gamma^{\frac{2}{a}} R_{1}^{2} \frac{2 \pi / a}{\sin (2 \pi / a)}}
$$

Therefore, we obtain

$$
\Pi_{3}=e^{-\gamma W R_{1}{ }^{a}} e^{-\pi \lambda_{c} \gamma^{\frac{2}{a}} R_{1}{ }^{2} \frac{2 \pi / a}{\sin (2 \pi / a)}},
$$

which concludes the proof.

\section{REFERENCES}

[1] ITU Report, “ICT Facts and Figures 2016," International Telecommunication Union, May 2016.

[2] J. Byun et al., "An intelligent self-adjusting sensor for smart home services based on ZigBee communications," IEEE Trans. Consumer Electronics, vol. 58, no. 3, pp. 794-802, August 2012.

[3] L. H. Wang et al., "An outdoor intelligent healthcare monitoring device for the elderly," IEEE Trans. Consumer Electronics, vol. 62, no. 2, pp. 128-135, May 2016.

[4] Newsletter 8 May 2015, "Nepal Earthquake," Fire and Rescue International, May 2015.

[5] H. Tabuchi, "Japan Panel Cites Failure in Tsunami," The New York Times, Dec. 2011.

[6] M. Benjamin, "Communications Breakdown," Salon, Sep. 2005.

[7] L. Xingqin et al., "An overview of 3GPP device-to-device proximity services," IEEE Commun. Mag., vol. 52, no. 4, pp. 40-48, Apr. 2014.

[8] L. Gupta, R. Jain and G. Vaszkun, "Survey of Important Issues in UAV Communication Networks," IEEE Commun. Surveys Tuts, vol. 18, no. 2, pp. 1123-1152, Secondquarter 2016.

[9] A. Al-Hourani et al., "Coverage and Rate Analysis of Aerial Base Stations," IEEE Trans. Aerosp. Electron. Syst., Oct. 2016.

[10] C. Zhang and W. Zhang, "Spectrum Sharing for Drone Networks," IEEE J. Sel. Areas Commun., vol. 35, no. 1, pp. 136-144, Jan. 2017.

[11] V. V. Chetlur Ravi and H. S. Dhillon, "Downlink coverage probability in a finite network of unmanned aerial vehicle (UAV) base stations," IEEE International Workshop on Signal Processing Advances in Wireless Communications (SPAWC), Edinburgh, 2016, pp. 1-5.

[12] Federal Communications Commission, "Part 15: Radio Frequency Devices," Code of Federal Regulations.

[13] J. G. Andrews et al., "A tractable approach to coverage and rate in cellular networks," IEEE Trans. Commun., vol. 59, no. 11, pp. 3122-3134, Nov. 2011.

[14] V. V. Chetlur, and H. S. Dhillon, "Downlink Coverage Analysis for a Finite 3D Wireless Network of Unmanned Aerial Vehicles", submitted, Jan. 2017. (arXiv).

[15] M. Mozaffari, W. Saad, M. Bennis, and M. Debbah, "Optimal transport theory for power-efficient deployment of unmanned aerial vehicles," in Proc. of IEEE International Conference on Communications (ICC), Kuala Lumpur, Malaysia, May. 2016.

[16] M. Peng, C. Wang, V. Lau and H. V. Poor, "Fronthaul-constrained cloud radio access networks: insights and challenges," IEEE Wireless Commun., vol. 22, no. 2, pp. 152-160, April 2015.

[17] D. Orfanus, E. P. de Freitas and F. Eliassen, "Self-Organization as a Supporting Paradigm for Military UAV Relay Networks," IEEE Commun. Letters, vol. 20, no. 4, pp. 804-807, April 2016.

[18] S. Schopferer, C. Liersch, M. Brizon and S. Froese, "Evaluating the energy balance of high altitude platforms at early design stages," Int. Conf. Unmanned Aircraft Systems (ICUAS), Arlington, VA, 2016, pp. 170-177.

[19] E. Bastug, M. Bennis and M. Debbah, "Living on the edge: The role of proactive caching in 5G wireless networks," IEEE Commun. Mag., vol. 52, no. 8, pp. 82-89, Aug. 2014. 
[20] T. Bai, R. Vaze and R. W. Heath, "Analysis of Blockage Effects on Urban Cellular Networks," IEEE Trans. Wireless Commun., vol. 13, no. 9, pp. 5070-5083, Sep. 2014.

[21] M. Haenggi, "Stochastic Geometry for Wireless Networks", 1st ed. Cambridge, U.K.: Cambridge Univ. Press, 2013.

[22] M. Afshang, H. S. Dhillon and P. H. Joo Chong, "Modeling and Performance Analysis of Clustered Device-to-Device Networks," IEEE Trans. Wireless Commun., vol. 15, no. 7, pp. 4957-4972, July 2016.

Prodromos-Vasileios Mekikis (S'13) received the degree in electrical and computer engineering from Aristotle University of Thessaloniki, Thessaloniki, Greece, and the M.Sc. degree in system-on-chip design from Royal Institute of Technology (KTH), Stockholm, Sweden, in 2010 and 2012, respectively. He is currently pursuing the Ph.D. degree at the Technical University of Catalonia (UPC), Barcelona, Spain. His research interests include wireless energy harvesting, and connectivity in dense wireless sensor networks.

Angelos Antonopoulos (S'10-M'12-SM'15) received the Ph.D. degree from Technical University of Catalonia (UPC), Barcelona, Spain, in 2012. His research interests include energy efficient network planning, cooperative communications, MAC protocols, and network coding. He received the Best Paper Award at the IEEE GLOBECOM in December 2014, and the Best Demo Award in the IEEE CAMAD, while in January 2015, he was nominated as an Exemplary Reviewer for the IEEE COMMUNICATIONS LETTERS.

Elli Kartsakli (S'07-M'09-SM'15) received the degree in electrical and computer engineering from the National Technical University of Athens, Athens, Greece, the M.Sc. degree in mobile and satellite communications from the University of Surrey, Surrey, U.K., and the Ph.D. degree from the Technical University of Catalonia (UPC), Barcelona, Spain, in 2003, 2004, and 2012, respectively. Her research interests include wireless networking, channel access protocols, and energy-efficient communication protocols.

Luis Alonso (S'99-AM'01-M'04-SM'13) received the Ph.D. degree from Technical University of Catalonia (UPC), Barcelona, Spain, in 2001. He was given a permanent tenured position at the UPC becoming an Associate Professor in 2006. He was a Co-Founder of the Wireless Communications and Technologies Research Group, WiComTec, UPC, to which he currently belongs. His research interests include medium access protocols, radio resource management, cross-layer optimization, cooperative transmissions, and cognitive radio.

Christos Verikoukis (S'95-AM'04-M'04-SM'07) received the Ph.D. degree from Technical University of Catalonia (UPC), Barcelona, Spain, in 2000. He is currently the Head of the SMARTECH Department, CTTC, Barcelona, Spain, and an Adjunct Professor with the University of Barcelona, Barcelona, Spain. He has authored 85 journal papers and over 160 conference papers. He is also a coauthor of three books, 14 chapters in other books, and two patents. He has participated in more than 30 competitive projects, and has served as a principal investigator of national projects in Greece and Spain. He is currently the Chair of the IEEE ComSoc Technical Committee on Communication Systems Integration and Modeling (CSIM). 